that of an out-pacient at Guy's Hospital. the illness which deprived us for a time of Dr. Hodgkin ordered dec. sarsa c. c. ex- your valuable instructions, we are desirous tract. Of course this article, prepared ac- of expressing to you our perfect satisfaccording to the Pharmacopoia Guyensis, tion with the lectures of your indefatigable which 1 presume, in this Institution, phy- and talented colleague IMr. Pilcher; and, sicians, surgeons, and pupils, expect the so far from concurring in the sentiments apothecary to be guided by, is a thick expressed in the attack which appeared in dark-coloured liquid, nearly black. But TME LANCET, we unanimously deprecate the trash given to the pationt was a straw- the conduct of the individual with whom coloured, sweetish, liquor, seemingly a de- it originated. In conclusion, we beg to coction of guaiacum and liquorice root, and, assure you of our complete satisfaction I am quite positive, did not contain a par- | with your constant and unwearied exerticle either of sarsaparilla or the extract. tions, in forwarding the interests of the From what I have seen during my stay in school at large, and subscribe ourselves; town, I do not believe that one half of the prescriptions written at this hospital are properly prepared. Certainly the physicians and surgeons, for their own characters' sake, for the benefit of the pupils, and particularly for the welfare of the pationts, ought occasionally to inspect the modicines they order. They could not knowingly allow themselves to be thus made fools of. If the apothecary be allowed to wink at the prescriptions, and, without knowing anything of the disease, to substitute what he pleases, we may dispense with the physicians altogether. The attendance, too, of the pupils is all a farce, leaving the simation of the poor patients out of the question. I am fully persuaded that you will not allow an abuse so pregnant with evil to us, and to the public at large, to go unexposed, and am, Sir, yours, \&c.,

March 14, 1833.

$$
\text { N,-A Pupil at GuY's. }
$$

\title{
Mr. GRAINGER.
}

\section{To the Editor of Tre LAxost.}

At a very numerous meeting of the members of Mr. Grainger's Surgical Class, held on the 27 th inst., after the surgical lecture, it was resolved unaninously, that an address, of which the following is a copy, should be immediately presented to Mr. Grainger, and that the proceeding. should be made public through the medium of THE LANCET.

\section{Danigl Noble,}

Chairman of the Meeting.

"To R. D. Grainger, Esq.-Sir, We, the undersigned pupils of the Surgical Class, indirnant at the appearance in TrE LANCET of the 23rd inst. of an anonymous and unjust attack upon your character, professedly originating with one of your pupils, are anxious to disclaim participation in any such act, and do hereby beg to inform you, that we consider the accusation of neglect of duty as totally false and untenable. Whilst, however, we regret

"Webb-street, March 27, 1833."

" (Here follow the signatures.)"

\section{INTERESTING PATHOLOGICAI FACTS.}

THe following is a fuller account, fur. nished by a correspondent, of the case of cedema confined to the upper half of the body, related by Mr. Beevor, of Berners Street, at the Middlesex Medical Society, on the 12th of March, and mentioned in THa LANCET, No. 491*:-

" The diagnosis was most faithfully delivered before the fatal termination of the case, to the following effect:-' Aneurism of the aorta, great disease of its membrane, and partial obstruction through the superior vena cava.' It should be stated, that the latter part of the diagnosis was fomded on the enormous turgidity and varicose condition of the veins over the chest, which inosculated freely with the epigastric veins, \&c., and thus Mr. Beevor conceived the abdomen was kept free from fluid, and the vessels relieved themselves by giving rise to cedema in the head and upper extremities, \&c., consequent on the impeded venous circulation near the aneurism. The man very rapidly sank, and, latterly, had very terrific notions about his sicuation, thinking he was among men of blood, ruurderers, thieves, \&c. This symptom was satisfactorily accounted for by the Chairman, from the mixture of arterial and venous blood in the following manner:-The aneurism had produced interstitial absorption of the sternum, and was capable of holding a pint of fluid; about an inch above the right auricle, the vena cara was quite impervious, to the extent of half an inch; and just above the obliteration was a rent of the coats, which led directly into the enlarged aorta beneath. The azygos on the one side, and the left subclavian, and a large peri-

* The Chairman on this occasion was Dr. F. Hawkins, and the gentleman who alluded to the cases of fever was $\not A r$, Corfe. 